\title{
Global Flows with Invariant (Gibbs) Measures for Euler and Navier-Stokes Two Dimensional Fluids
}

\author{
Sergio Albeverio ${ }^{1}$ and Ana-Bela Cruzeiro ${ }^{2}$ \\ ${ }^{1}$ Fakultät für Mathematik, Ruhr-Universität-Bochum, D-4630 Bochum, BiBoS Research Centre and \\ SFB 237 Bochum-Essen-Düsseldorf, Federal Republic of Germany \\ ${ }^{2}$ Centro de Matemática e Aplicações Fundamentais, I.N.I.C., Av. Prof. Gama Pinto, 2, P-1699 Lisboa, \\ Codex, Portugal
}

\begin{abstract}
We construct a family of probability spaces $\left(\Omega, \mathscr{F}, P_{\gamma}\right), \gamma>0$ associated with the Euler equation for a two dimensional inviscid incompressible fluid which carries a pointwise flow $\phi_{t}$ (time evolution) leaving $P_{\gamma}$ globally invariant. $\phi_{t}$ is obtained as the limit of Galerkin approximations associated with Euler equations. $P_{\gamma}$ is also in invariant measure for a stochastic process associated with a Navier-Stokes equation with viscosity $\gamma$, stochastically perturbed by a white noise force.
\end{abstract}

\section{Contents}

0. Introduction . . . . . . . . . . . . . . . . . . . . 431

1. The Euler Equation in Two Dimensions. . . . . . . . . . . 433

1.1. General Setting . . . . . . . . . . . . . . . . . . 433

1.2. Invariant Quantities of the Motion . . . . . . . . . . . 434

1.3. The Abstract Wiener Space Formulation . . . . . . . . . 434

2. The Euler Flow and the Invariant Measures . . . . . . . . . 437

2.1. The Invariant Measures . . . . . . . . . . . . . . . 437

2.2. The Euler Flow. . . . . . . . . . . . . . . . . . 438

3. The Perturbed Navier-Stokes Equation. . . . . . . . . . . 440

3.1. Invariant Measures . . . . . . . . . . . . . . . . 440

3.2. The Perturbed Navier-Stokes Flow. . . . . . . . . . . 441

\section{Introduction}

The search for solutions of Euler and Navier-Stokes equations in certain spaces of functions is of great physical and mathematical interest. In the present paper we shall discuss the Euler and Navier-Stokes equations for an incompressible fluid.

For simplicity we shall consider a fluid confined in a rectangular box $\mathbb{T}^{2}$, with periodic boundary conditions (or, equivalently, on a 2-torus), but extensions to 
more general domains are possible. The equations of motion are

$$
\frac{\partial u}{\partial t}=-(u \cdot \nabla) u-\nabla p+\gamma \Delta u, \quad \operatorname{div} u=0,
$$

where $u(t, x)=\left(u_{1}(t, x), u_{2}(t, x)\right) \in \mathbb{R}^{2}, t \in \mathbb{R}, x \in \mathbb{T}^{2}=[0,2 \pi] \times[0,2 \pi]$ is the velocity field, $\gamma \geqq 0$ is a constant coefficient, $u \cdot \nabla \equiv u_{1}\left(\partial / \partial x_{1}\right)+u_{2}\left(\partial / \partial x_{2}\right) \equiv u_{1} \partial_{1}+u_{2} \partial_{2}$, and $\Delta$ is the Laplacian in $\mathbb{R}^{2}, \operatorname{div} u \equiv \sum_{i=1}^{2} \partial_{i} u_{i}, \nabla p \equiv\left(\partial_{1} p, \partial_{2} p\right)$. div $u=0$ is the incompres-
sibility condition.

Concerning such equations (on $\mathbb{T}^{2}$ or domains in $\mathbb{R}^{2}$ ) two types of results are known:

a) results on generalized and pointwise classical solutions, with initial data in some (generalized) function spaces (not necessarily "physically relevant")

b) results on statistical solutions, with initial data in some measure one set in a probability space describing "physical initial conditions."

Concerning a) let us mention that in the case $\gamma=0$ global (in time) solutions with initial data with finite energy are known (M.M. Gunther (1927), W. Wolibner (1933), V. Judovich (1963), T. Kato (1967), C. Bardos (1972), see e.g. the references in $[\mathrm{E}])$.

In the case $\gamma>0$, we mention the existence of global solutions by J. Leray (1933), O. Ladyszenskaja (1959), Lions-Prodi (1959), see e.g. [Te] and references therein.

Concerning b): this is part of a statistical approach to turbulence, initiated already last century, see e.g. [MP] and references therein. For the particular two-dimensional situation we are considering the case $\gamma=0$ has been discussed in physical literature by T. D. Lee (1952), R. H. Kraichnan (1967) and others, see e.g. [G], [KrMo]. Probability measures $\mu$ of Gibbsian type, with Gibbs density determined by the invariants of the motions, have been discussed mathematically in $[\mathrm{AHKDeF}],[\mathrm{AHK} 1],[\mathrm{DeF}],[\mathrm{AHKM}],[\mathrm{BF} 1,2],[\mathrm{BPP}],[\mathrm{CDG}]$. Their "infinitesimal invariance" in the sense $\int B f d \mu=0$ for smooth cylinder functions in the domain of the corresponding Liouville operator, has been proven in [AHKDeF], [AHK1]. As to the existence of a global flow in time leaving $\mu$ invariant, only partial results had been obtained in the case of perturbed Gaussian measures [AHK1], [AHKM], [DeF], [CDG] and in the case of Poisson measure [DP], [MP]. For $\gamma>0$ statistical solutions of different types have been discussed, see e.g. $[\mathrm{VKF}],[\mathrm{FT}]$ and references therein. However to our knowledge, the only result on invariant probability measures which has been proven in the case $\gamma>0$ needs the presence of an additional suitable random force $[\mathrm{C} 1,2]$.

In Sects. 1 and 2 of the present paper we give the first proof that the infinitesimally invariant probability measures constructed in [AHKDeF], [AHK1], $[\mathrm{DeF}],[\mathrm{BF} 1,2],[\mathrm{BPP}],[\mathrm{CDG}]$ are indeed globally invariant. In Sect. 3 we extend the result to a stochastically perturbed Navier-Stokes equation.

Let us now describe the contents of the different sections in more details. In Sect. 1 we discuss the equivalence of the Euler equation for a fluid on a 2-dimensional torus (rectangular box with periodic boundary conditions) with an equation for a scalar "stream function" $\varphi$. Moreover we express the basic conserved 
quantities of the (classical, smooth) Euler flow, energy and enstrophy, in terms of $\varphi$. Finally we introduce the basic family $\mu_{\gamma}, \gamma>0$ of heuristically invariant probability measures of the Gibbs form, with formal density given by the enstrophy (a measure giving, roughly speaking, white noise distribution to the curl of the velocity vector). These probability measures are defined in terms of an abstract (complex) Wiener space, a complex version of a setting studied particularly by Gross, cf. e.g. [K]). A basic Lemma 1.3.2 shows that the generator $B$ associated with the Euler equation (looked upon as an evolution equation in the Fourier transformed space to the stream function) is in $L^{2}$ between basic Sobolev spaces $H^{1-\alpha}, \alpha>\frac{3}{2}$ with respect to the basic probability measure $\mu_{\gamma}$.

In Sect. 2 we show that $B$, as a functional with values in $H^{1-\alpha}$, is divergence free (relative to the divergence defined in the abstract Wiener space). In fact the latter result and the mentioned Lemma 1.3.2 essentially express in the useful language of abstract Wiener spaces results contained in [AHKDeF]. The usefulness of this formulation becomes clear in 2.2 where the basic Theorem 2.2.3 on the existence of a continuous flow defined on a probability space $\left(\Omega, \mathscr{F}, P_{\gamma}\right)$ with values in $H^{1-\alpha}$, associated to the Euler equation and leaving $\mu_{\gamma}$ globally invariant, is stated and proven. As mentioned before, this is the first solution of a problem stated in [AHKDeF] and [BF] (see also [AHKM], [CDG] which contain partial results in this direction). It should be remarked that only existence is shown, the problem of uniqueness remains open, see [AHKM], [AHK1] for a discussion of this problem.

Let us also remark that the present solution of the problem of constructing a pointwise flow should not be confused with the construction of a flow in $L^{2}$-sense: the latter flow had been already constructed in [AHKDeF] (see also [AHK1], [AHKM] for further discussion). Of course the present pointwise flow also yields an $L^{2}$ flow. The problem of uniqueness of $L^{2}$ flows remains open (cf. [AHK1], [AHKM]).

Finally let us remark that using results of [AHKDeF] (see also [AHK1], [AHKM], [W]) all is said here about $\mu_{\gamma}, P_{\gamma}$, can be extended to measures $\mu_{\beta, \gamma}, P_{\beta, \gamma}$ constructed from $\mu_{\gamma}$ using the renormalized energy functional : $E_{\gamma}$ : of [AHKDeF], i.e. $d \mu_{\beta, \gamma}=\exp \left(-\beta: E:_{\gamma}\right) d \mu_{\gamma} / \int \exp \left(-\beta: E:_{\gamma}\right) d \mu_{\gamma}$ with $\beta \geqq 0$ (cf. [W]). We have $\mu_{\beta, \gamma} \ll \mu_{\beta^{\prime}, \gamma}$ $\forall \beta, \beta^{\prime}, \gamma, \mu_{\beta, \gamma} \perp \mu_{\beta, \gamma^{\prime}}, \gamma \neq \gamma^{\prime}$. In particular $P_{\beta, \gamma}$ is a family of globally invariant probability measures associated with the Euler flow.

In Sect. 3 we consider the stochastically perturbed Navier-Stokes equation. In 3.1 we show that each invariant measure $\mu_{\gamma}$ for the Euler flow is also an invariant measure for a certain infinite dimensional Ornstein-Uhlenbeck process. Using this in Sect. 3.2 it is shown (Theorem 3.2.1) that $\mu_{\gamma}$ is also an invariant measure for the flow associated with a Navier-Stokes equation perturbed by a suitable external random force.

\section{The Euler Equation in Two Dimensions}

1.1. General Setting. Let us consider the Euler equation for an incompressible non-viscous fluid in $\mathbb{R}^{2}$, given by

$$
\frac{\partial u}{\partial t}=-(u \cdot \nabla) u-\nabla p, \quad \operatorname{div} u=0,
$$


where $u$ is the velocity of the flow, $p$ the pressure, $u \cdot \nabla=u_{1} \partial_{1}+u_{2} \partial_{2}$. We will consider the Euler equation on the 2-dimensional torus that we identify with the square $\mathbb{T}^{2}=[0,2 \pi] \times[0,2 \pi]$ and with periodic boundary conditions. Let rot denote the operator $\operatorname{rot} u=-\partial_{2} u^{1}+\partial_{1} u^{2}$ (for $u^{i} \in C^{1}$ ) and set $\nabla^{\perp} \varphi \equiv\left(-\partial_{2} \varphi, \partial_{1} \varphi\right.$ ), where $\varphi$ is a scalar $C^{2}$ function. The incompressibility condition ( $\left.\operatorname{div} u=0\right)$ implies the existence of a function $\varphi$ such that $u=\nabla^{\perp} \varphi$. Replacing in the Euler equation $u$ by $\nabla^{\perp} \varphi$ and applying the operator rot to both members, one can find a different formulation of the equation, expressed by the following result ([AHKDeF]):

1.1.1. Theorem. $u$ is a smooth solution of (1.1) if and only if there exists a smooth (real) function $\varphi$ such that $u=\nabla^{\perp} \varphi$ and $\varphi$ is a solution of the equation

$$
\frac{\partial \Delta \varphi}{\partial t}=\nabla^{\perp} \varphi \cdot \nabla \Delta \varphi
$$

We are therefore interested in solutions of Eq. (1.2) on $\mathbb{T}^{2}$ satisfying the periodic boundary conditions

$$
\varphi(0, y, t)=\varphi(2 \pi, y, t) \quad \text { and } \quad \varphi(x, 0, t)=\varphi(x, 2 \pi, t), \quad \forall(x, y) \in \mathbb{T}^{2} .
$$

Remark. Considering, on a bounded domain $D \subset \mathbb{R}^{2}$ with simply connected piecewise $C^{1}$ boundary, the Euler equation $\partial u / \partial t=-(u \cdot \nabla) u-\nabla p$, div $u=0$, with $n \cdot u=0$ on $\partial D$, where $n$ is a unit normal to $\partial D$, we still have a formulation analogous to (1.2). See [AHK1] for a study of this situation, as well as for the study of the Euler equations in more general domains, also in connection with the content of the other sections in the present paper.

1.2. Invariant Quantities of the Motion. As is well known, the Euler system is conservative, that is, the energy is an invariant of the motion. This is easily seen by looking at the energy in terms of $\varphi \in C^{2}\left(\mathbb{T}^{2}, \mathbb{R}\right)$,

$$
E=\frac{1}{2} \int_{\mathbb{J}^{2}} u^{2} d x=-\frac{1}{2} \int_{\mathbb{T}^{2}} \varphi \Delta \varphi d x .
$$

We have for any smooth solution $\varphi$ of (1.2):

$$
\frac{d E}{d t}=-\int_{\mathbb{T}^{2}} \varphi \nabla^{\perp} \varphi \nabla \Delta \varphi d x=\int_{\mathbb{T}^{2}} \nabla \varphi \nabla^{\perp} \varphi \Delta \varphi d x=0 .
$$

In a similar way, one can see that there are other invariant quantities for the Euler system (cf. [AHKDeF]). One such quantity that will be important for our purposes is the so-called "enstrophy," given by:

$$
S=\frac{1}{2} \int_{\mathbb{J}^{2}}(\operatorname{rot} u)^{2} d x=\frac{1}{2} \int_{\mathbb{T}^{2}}(\Delta \varphi)^{2} d x .
$$

We have in fact, for any $C^{2}$ solution $\varphi$ of (1.2):

$$
\frac{d S}{d t}=\int_{\mathbb{J}^{2}} \Delta \varphi \nabla^{\perp} \varphi \nabla \Delta \varphi d x=0 .
$$

1.3. The Abstract Wiener Space Formulation. Let us consider the following 
Sobolev-space on the torus:

$$
\mathscr{H}^{2}\left(\mathbb{T}^{2}\right)=\left\{u: \mathbb{T}^{2} \rightarrow \mathbb{R}: \int \sum_{|\alpha| \leqq 2}\left|D^{\alpha} u(x)\right|^{2} d x<+\infty\right\} .
$$

Let $e_{k}(x)=(1 / 2 \pi) e^{i k \cdot x}$, with $k \in \mathbb{Z}^{2}$ and $k \cdot x=k_{1} x_{1}+k_{2} x_{2}$, be a complete set of orthonormal (with respect to $L^{2}$ ) functions, which are eigenfunctions of the operator $-\Delta$, having $k^{2}=k_{1}^{2}+k_{2}^{2}$ as their eigenvalues. We can identify $\mathscr{H}^{2}\left(\mathbb{T}^{2}\right)$ with the complex Hilbert space

$$
H^{2}=\left\{u=\sum u_{k} e_{k}: \sum k^{4}\left|u_{k}\right|^{2}<+\infty, u_{-k}=\bar{u}_{k}\right\}
$$

(with $\bar{u}$ denoting complex conjugation of $u$ ).

For general $p \in \mathbb{R}$ we define:

$$
H^{p}=\left\{u=\sum_{k>0} u_{k} e_{k}: \sum k^{2 p}\left|u_{k}\right|^{2}<+\infty\right\}
$$

( $k>0$ meaning $k \in \mathbb{Z}^{2}, k_{1}>0$ or $k_{1}=0$ and $k_{2}>0$ ), and with inner product given by $\langle u, v\rangle_{p}=\sum_{k>0} k^{2 p} u_{k} \bar{v}_{k}$.

Let $d \mu_{\gamma}^{k}$ be the probability measure on $\mathbb{C}$ defined for $\gamma \in \mathbb{R}^{+}$by

$$
d \mu_{\gamma}^{k}(z)=\frac{\gamma k^{4}}{2 \pi} \exp \left(-\frac{1}{2} \gamma k^{4}|z|^{2}\right) d x d y
$$

where $z=x+i y$, and $d \mu_{\gamma}(u)=\prod_{k>0} d \mu_{\gamma}^{k}\left(u_{k}\right)$. It is easy to see that, for $\alpha>0$, $\int\|u\|_{1-\alpha}^{2} d \mu=\int \sum_{k>0} k^{2-2 \alpha}\left|u_{k}\right| d \mu_{\gamma}(u)=\frac{2}{\gamma} \sum_{k>0} \frac{1}{k^{2+2 \alpha}}<+\infty$. So the measure $\mu_{\gamma}$ is supported by $H^{1-\alpha}$.

We shall call complex abstract Wiener measure space a complex structure consisting of a complex Hilbert space $H$, a complex Banach space $B$ which is the completion of $H$ with respect to a measurable norm in the sense of Gross (cf. $[\mathrm{K}]$ ) and a $\sigma$-additive normalized measure on $B$. We claim that:

1.3.1. Proposition. $\left(H^{1-\alpha}, H^{2}, \mu_{\gamma}\right)$ is a complex abstract Wiener space with measurable norm $\|\cdot\|_{1-\alpha}$, for any $\alpha>0$.

Proof. Let $A$ be the linear operator defined by $A\left(e_{k}\right)=\left(1 /|k|^{1+\alpha}\right) e_{k} . A$ is an Hilbert-Schmidt operator on $H^{2}$ with norm $\|A\|_{\text {H.S. }}^{2}=\sum_{k} \frac{1}{k^{2(1+\alpha)}}<+\infty$. For $u=\sum u_{k} e_{k} \in H^{2}$ we have $\|A(u)\|_{2}=\|u\|_{1-\alpha}$. The norm $\|\cdot\|_{1-\alpha}$ is then a measurable norm and the completion of $H^{2}$ with respect to it gives the space $H^{1-\alpha}$.

Remark. In the framework of the remark of paragraph 1.1, and considering $\Delta$ to be the Laplacian with Dirichlet boundary conditions, one knows that $-\Delta$ has a discrete spectrum $0<\lambda_{1} \leqq \cdots \leqq \lambda_{i} \cdots, \lambda_{i} \rightarrow+\infty$. We can therefore make an analogous Wiener space formulation in this context.

The measure $\mu_{\gamma}$ constructed above is then the Wiener measure for the space 
$H^{1-\alpha}$, verifying:

$$
\int \exp (i \gamma l(u)) d \mu_{\gamma}(u)=\exp \left(-\frac{1}{2}\|\gamma l\|_{2}^{2}\right) \quad \forall l \in\left(H^{1-\alpha}\right)^{*} \subset H^{2} .
$$

We have $E_{\mu_{\gamma}}\left(u_{k}\right)=0, E_{\mu_{\gamma}}\left(u_{k} u_{k}^{\prime}\right)=0$ and $E_{\mu_{\gamma}}\left(u_{k} \bar{u}_{k}^{\prime}\right)=\delta_{k, k^{\prime}}\left(2 / \gamma k^{4}\right)$. Moreover for $\gamma \neq \gamma^{\prime}$ the measures $\mu_{\gamma}$ and $\mu_{\gamma^{\prime}}$ are orthogonal. Furthermore we have $\mu_{\gamma}\left(H^{2}\right)=0$.

On the abstract Wiener space constructed above we will use differential calculus in the sense of Malliavin (see [Ma] for an introduction). In particular we shall use derivatives along the directions belonging to the Cameron-Martin space, that is, $H^{2}$.

Let us come back to the Euler equation (1.2) and write $\varphi(x, t)=\sum_{k>0} u_{k}(t) e_{k}$ ( $\varphi$ is real and we can assume $\int \varphi d x=0$ since adding a constant to $\varphi$ does not change Eq (1.2)). Now (1.2) takes the form (cf. [AHKDeF]):

$$
\begin{aligned}
2 \pi k^{2} \frac{d}{d t} u_{k} & =-\sum_{h+h^{\prime}=k}\left(h^{\perp} \cdot h^{\prime}\right)\left(h^{\prime}\right)^{2} u_{h} u_{h^{\prime}} \\
& =\frac{1}{2} \sum_{h+h^{\prime}=k}\left(h^{\perp} \cdot h^{\prime}\right)\left[h^{2}-\left(h^{\prime}\right)^{2}\right] u_{h} u_{h^{\prime}},
\end{aligned}
$$

where $h^{\perp}=\left(-h_{2}, h_{1}\right)$. This can be written as

$$
\frac{d}{d t} u_{k}=B_{k}(u)
$$

where the $B_{k}$ are given by:

$$
B_{k}(u)=\frac{1}{2 \pi} \sum_{h}\left[\frac{1}{k^{2}}\left(h^{\perp} \cdot k\right)(h \cdot k)-\frac{1}{2}\left(h^{\perp} \cdot k\right)\right] u_{h} u_{k-h},
$$

and we look for solutions $u(t) \in H^{1-\alpha} \forall t$. Let $B(u)=\sum_{k} B_{k}(u) e_{k}$. Then we have:

1.3.2. Lemma. For every $\alpha>\frac{3}{2}$ the functional $B$ is square-integrable with respect to the measure $\mu_{\gamma}$ and to the norm of $H^{1-\alpha}$, i.e.,

$$
E_{\mu_{\gamma}}\|B\|_{1-\alpha}^{2}<+\infty \text {. }
$$

Proof. The proof can be found in [AHKDeF]; nevertheless, we sketch it here, for sake of completeness. Define the following approximation fields:

$$
B_{k}^{n}(u)=\sum_{h^{2} \leqq n} \alpha_{h, k} u_{h} u_{k-h},
$$

where $2 \pi \alpha_{h, k}=\left(1 / k^{2}\right)\left(h^{\perp} k\right)(h k)-\frac{1}{2}\left(h^{\perp} k\right)$. Then we have

Hence,

$$
\begin{aligned}
E_{\mu_{\gamma}}\left(\left|B_{k}^{n}\right|^{2}\right)= & \sum_{\substack{h^{2} \leqq n \\
\left(h^{\prime}\right)^{2} \leqq n}}\left(\alpha_{h, k} \alpha_{h^{\prime}, k}\right) E\left(u_{h} u_{k-h} \bar{u}_{h^{\prime}} \bar{u}_{k-h^{\prime}}\right) \\
= & \frac{4}{\gamma^{2}} \sum_{\substack{h^{2}<n \\
h^{\prime 2} \leqq n}} \alpha_{h, k} \alpha_{h^{\prime}, k}\left(\delta_{h, h^{\prime}}+\delta_{h, k-h^{\prime}}\right) \cdot \frac{1}{h^{4}(k-h)^{4}} .
\end{aligned}
$$

$$
E_{\mu_{\gamma}}\left(\left|B_{k}^{n}\right|^{2}\right) \leqq \frac{2}{(\pi \gamma)^{2}} \sum_{h \neq k} \frac{1}{(k-h)^{4}}+\frac{1}{2(\pi \gamma)^{2}} \sum_{h \neq k} \frac{k^{2}}{h^{2}(k-h)^{4}}
$$




$$
+\frac{1}{(\pi \gamma)^{2}} \sum_{h \neq k} \frac{1}{|h|^{3}|k-h|^{3}}\left\{|k-h|+\frac{1}{2}|k|\right\}\left\{|h|+\frac{1}{2}|k|\right\},
$$

and, for $m<n, E_{\mu_{\gamma}}\left(\left|B_{k}^{n}-B_{k}^{m}\right|^{2}\right)$ is estimated by the r.h.s. of (1.6) with summations restricted to $m^{2}<h^{2} \leqq n^{2}$, which proves that $B_{k}^{n}$ is a Cauchy sequence in $L^{2}\left(H^{1-\alpha}, \mathbb{C}\right)$, converging to $B_{k}$. Finally, for $\alpha>\frac{3}{2}$ we have $E_{\mu_{\nu}}\left(\sum_{k} k^{2-2 \alpha}\left|B_{k}\right|^{2}\right)<+\infty$, and therefore $B \in L^{2}\left(H^{1-\alpha}, H^{1-\alpha}\right)$.

We remark that $B$ is not a vector field in the sense of [C3], that is, it is not a functional with values in the Cameron-Martin space. Therefore one cannot expect to apply the results of [C3] to construct a flow associated with $B$.

We also remark that the energy and the enstrophy defined in 1.2 are given by:

$$
E=\frac{1}{2} \sum_{k} k^{2}\left|u_{k}\right|^{2}=\frac{1}{2}\|u\|_{1}^{2}, \quad S=\frac{1}{2} \sum_{k} k^{4}\left|u_{k}\right|^{2}=\frac{1}{2}\|u\|_{2}^{2} \text {. }
$$

\section{The Euler Flow and the Invariant Measures}

2.1. The Invariant Measures. Let us consider the gradient operator in the sense of the Malliavin calculus, that is, for $\varphi$ defined on the Wiener space $H^{1-\alpha}$ with values in a Banach space $E, \nabla \varphi(u)$ is the linear functional defined, for $u \in H^{1-\alpha}$, by:

$$
\nabla \varphi(u)(v)=D_{v} \varphi(u)=\lim _{\varepsilon \rightarrow 0} \frac{1}{\varepsilon}[\varphi(u+\varepsilon v)-\varphi(u)], \quad v \in H^{2} .
$$

Although, as we remarked, $B$ is not a vector field on the Wiener space, we shall consider the following generalization of the notion of divergence (cf., for example, [KK]).

2.1.1. Definition. If $G$ is a Hilbert space and $\varphi \in L^{2}\left(H^{1-\alpha}, G\right) \equiv L^{2}$, we call divergence of $\varphi$, and we denote it by $\delta_{\mu} \varphi$, the adjoint of $\nabla$ in $L^{2}$, i.e., $\delta_{\mu} \varphi$ is the element of $L^{2}\left(H^{1-\alpha}, \mathbb{R}\right)$, which verifies, whenever it exists:

$$
\int \delta_{\mu} \varphi \cdot f d \mu=\int(\varphi \mid \nabla f)_{G} d \mu \quad \forall f \in \mathscr{D},
$$

where $\mathscr{D}$ is the space of differentiable functions defined on $H^{1-\alpha}$ and depending only on a finite number of coordinates $u_{k}$. $\mathscr{D}$ will be used as the space of test functions. $(\mid)_{G}$ is the scalar product in $G$.

2.1.2. Lemma. Considering $B$ as given in Sect. 1.3, as a functional with values in $H^{1-\alpha}, \alpha>\frac{3}{2},\left(G=H^{1-\alpha}\right.$ in definition 2.1.1) we have $\delta_{\mu_{\gamma}} B=0$, with $\mu_{\gamma}$ as in 1.3.

Proof (cf. [AHKDeF]). Consider the Galerkin approximations $B_{k}^{n}$ of $B_{k}$ defined by (1.5); $B_{k}^{n}$ depends only on a finite number of coordinates, say $\left(u_{\alpha_{1_{k}}}, \ldots, u_{\alpha_{d_{k}}}\right)$, where $\alpha_{i} \in \mathbb{Z}^{2}, d_{k}=d_{k}(n)$. By taking $B_{1}^{n}, \ldots, B_{n}^{n}$, we can define:

where $d=d(n)$.

$$
B^{n}=\sum_{k \in\left\{\alpha_{1}, \ldots, \alpha_{d}\right\}} B_{k}^{n} e_{k}
$$

$B^{n}$ is then a vector field on $\mathbb{C}^{d}$ and we know that, with respect to the measure

$$
d \mu_{\gamma}^{n}(u)=\prod_{k \in\left\{\alpha_{1}, \ldots, \alpha_{d}\right\}} d \mu_{\gamma}^{k}(u), \quad u=\left(u_{\alpha_{1}}, \ldots, u_{\alpha_{d}}\right)
$$


the divergence is given by

$$
\delta_{\mu_{\gamma}^{n}} B^{n}(u)=\left\langle B^{n}(u), u\right\rangle_{2}-\sum_{k \in\left\{\alpha_{1}, \ldots, \alpha_{d}\right\}} D_{e_{k}} B_{k}^{n}(u) .
$$

From the expression (1.3) we see that, for each $k, D_{e_{k}} B_{k}^{n}=0$. On the other hand,

$$
\left\langle B^{n}(u), u\right\rangle_{2}=\sum k^{4} B_{k}^{n}(u) \bar{u}_{k}=\left\langle\Delta B^{n}(u), \Delta u\right\rangle_{0}=0
$$

by the invariance of the enstrophy (cf. 1.2). Therefore we have $\delta_{\mu_{\gamma}^{n}} B^{n}=0 \forall n$ and the result follows.

According to [AHKDef], all measures $\mu_{\gamma}$ are infinitesimally invariant under the Euler flow in the sense that $\int B f d \mu_{\gamma}=0 \forall f \in \mathscr{D}$. The existence of a global Euler flow was left open in $[\mathrm{AHKDeF}]$. The purpose in the next section is to prove the existence of such a flow for $\mu_{\gamma}$-almost all initial conditions.

We remark that:

$$
E_{\mu_{\gamma}}\left(\sum_{k^{2} \leqq n} k^{2}\left|u_{k}\right|^{2}\right)=\frac{2}{\gamma} \sum_{k^{2} \leqq n} \frac{1}{k^{2}} \rightarrow+\infty \quad \text { as } n \rightarrow \infty .
$$

Therefore the energy is not in $L^{1}\left(\mu_{\gamma}\right)$ and it is not possible, in order to construct a flow, to use results on existence of solutions of the Euler equation with initial conditions of finite energy.

It is shown in [AHKDeF] that $\sum_{k^{2} \leqq n} k^{2}\left|u_{k}\right|^{2}-E_{\mu_{\nu}}\left(\sum_{k^{2} \leqq n} k^{2}\left|u_{k}\right|^{2}\right)$ converges in $L^{2}\left(\mu_{\gamma}\right)$ for $n \rightarrow \infty$. The limit is the "renormalized energy" discussed in [AHKDeF], [AHK1], [AHKM].

\subsection{The Euler Flow}

2.2.1 Lemma. There exists a unique non-explosive flow $u^{n}(0) \rightarrow u^{n}(t)$, with $(d / d t) u^{n}=$ $B^{n}(u)$ associated with the finite dimensional vector field $B^{n}$ defined in (1.5), (2.1).

Proof. Since $B_{k}^{n}$ is a finite sum of quadratic expressions of the type $\alpha_{h, k} u_{h} u_{k-h}$, this follows from the classical results on finite-dimensional flows. We remark that the non-explosion (in finite time) can also be proved using the fact that $B^{n}$ is divergence free (cf. [C3]).

We shall show that this classical flow (with support on functions) can be used to construct a flow (with support on generalized functions) associated with $B$.

For $u=\sum u_{k} e_{k} \in H^{1-\alpha}$, let $\Pi_{n} u$ be the orthogonal projection of $u$ on the subspace generated by $\left\{e_{\alpha_{1}}, \ldots, e_{\alpha_{d}}\right\}$. Write $u=\Pi_{n} u+\Pi_{n}^{\perp} u$ and let $U^{n}$ be the flow associated with $B^{n}$. Putting $\tilde{U}^{n}(t, u)=U^{n}\left(t, \Pi_{n} u\right)+\Pi_{n}^{\perp} u$, we have:

$$
\frac{d}{d t} \tilde{U}^{n}(t, u)=B^{n}\left(\tilde{U}^{n}(t, u)\right), \quad \tilde{U}^{n}(0, u)=u
$$
these flows being now defined on $H^{1-\alpha}$. Furthermore, if $\tilde{U}^{n}(\cdot, u)=\sum_{k} \tilde{U}_{k}^{n}(\cdot, u) e_{k}$, we
have that, for each $k, \tilde{U}_{k}^{n}(\cdot, u) \in C(\mathbb{R} ; \mathbb{C})$.

We are now able to show the existence of a flow (in the precise sense of the following result): 
2.2.3. Theorem. There exists a flow $U(t, \omega)$ defined on a probability space $\left(\Omega, \mathscr{F}, P_{\gamma}\right)$ with values in $H^{1-\alpha}, \alpha>\frac{3}{2}, U(\cdot, \omega) \in C\left(\mathbb{R} ; H^{1-\alpha}\right), \omega \in \Omega$, such that:

$$
U_{k}(t, \omega)=U_{k}(0, \omega)+\int_{0}^{t} B_{k}(U(s, \omega)) d s, \quad P_{\gamma} \text { - a.e. } \omega, \quad \forall t \in \mathbb{R},
$$

and such that the measure $\mu_{\gamma}$ is invariant for the flow, in the sense that:

$$
\int f(U(t, \omega)) d P_{\gamma}(\omega)=\int f d \mu_{\gamma} \quad \forall t, \forall f \in \mathscr{D} .
$$

Proof. We shall consider, for $t \in \mathbb{R}^{+}$, the $\tilde{U}_{k}^{n}$ as stochastic processes with law on $C(\mathbb{R} ; \mathbb{C})$ defined by

$$
v_{k}^{n}(\Gamma)=\mu_{y}\left(\left\{u: \tilde{U}_{k}^{n}(\cdot, u) \in \Gamma\right\}\right), \quad \Gamma \subset C\left(\mathbb{R}^{+} ; \mathbb{C}\right) .
$$

We consider the sup-norm on the space $C\left(\mathbb{R}^{+} ; \mathbb{C}\right)$ and the weak topology on the space of measures over $C\left(\mathbb{R}^{+} ; \mathbb{C}\right)$. If we verify the following conditions:

$$
\begin{aligned}
& \lim _{R \rightarrow+\infty} \sup _{n} v_{k}^{n}(|y(0)|>R)=0, \\
& \lim _{\delta \rightarrow 0} \sup _{n} v_{k}^{n}\left(\sup _{\substack{0 \leqq t \leqq t^{\prime} \leqq r \\
t^{\prime}-t \leqq \delta}}\left|y(t)-y\left(t^{\prime}\right)\right| \geqq \rho\right)=0 \quad \forall \rho>0, \quad T>0,
\end{aligned}
$$

then it follows, by Prokhorov's criterium (cf., for example, [SV]) that $v_{k}^{n}$ is a precompact sequence of measures and therefore that we have a subsequence converging weakly towards a limit $v_{k}$. We have:

which implies (a);

$$
v_{k}^{n}(|y(0)|>R) \leqq \frac{1}{R^{2}} E_{\mu_{\gamma}}\left(\left|u_{k}\right|^{2}\right) \leqq \frac{2}{\gamma R^{2} k^{2}},
$$

(b) comes from the following estimations:

$$
\begin{aligned}
v_{k}^{n}\left(\sup _{t, t^{\prime}}\left|y(t)-y\left(t^{\prime}\right)\right| \geqq \rho\right) & \leqq \frac{1}{\rho^{2}} E_{\mu_{\gamma}}\left(\sup \left|\tilde{U}_{k}^{n}(t, u)-\tilde{U}_{k}^{n}\left(t^{\prime}, u\right)\right|^{2}\right) \\
& \leqq \frac{\delta}{\rho^{2}} E_{\mu_{\gamma}} \int_{0}^{T}\left|B_{k}^{n}\left(U^{n}(s, u)\right)\right|^{2} d s \\
& \leqq \frac{\delta}{\rho^{2}} T E_{\mu_{\gamma}}\left(\left|B_{k}^{n}\right|^{2}\right) \leqq C \frac{\delta T}{\rho^{2}}
\end{aligned}
$$

where we used (1.6) and the fact that $\mu_{\gamma}$ is invariant for the approximating $U^{n}$ flows (the divergence of $B^{n}$ is zero).

There is then a subsequence of $v_{k}^{n}$ converging to $v_{k}$; we shall denote it again by $v_{k}^{n}$, for simplicity. By Skorohod's theorem (cf., for example [IW]), there exists a probability space $\left(\Omega, \mathscr{F}, P_{\gamma}\right)$ and a family of processes $\widetilde{U}_{k}^{\prime \prime}(t, \omega), U_{k}(t, \omega), \omega \in \Omega$, having for laws, respectively, $v_{k}^{n}$ and $v_{k}$ on the space $C\left(\mathbb{R}^{+} ; \mathbb{C}\right)$. Furthermore, $\tilde{U}_{k}^{\prime n}(\cdot, \omega) \rightarrow U_{k}(\cdot, \omega) P_{\gamma}-$ a.e. in $\omega$. On the other hand, we can repeat this construction for the processes $t \in \mathbb{R}^{+} \mapsto \tilde{U}_{k}^{n}(-t, u)$ to obtain the negative values of $t$.

We first prove (ii); for $f \in \mathscr{D}$, we have:

$$
\int f\left(\tilde{U}^{n}(t, u)\right) d \mu_{\gamma}(u)=\int d \mu_{\gamma}^{n, \perp} \int f\left(U_{t}^{n}(u)\right) d \mu_{\gamma}^{n}(u) \quad \forall t,
$$

where $d \mu_{\gamma}^{n}$ is defined in $(2.2)$ and $d \mu_{\gamma}^{n, \perp}(u)=\prod_{k \notin\left\{\alpha_{1}, \ldots, \alpha_{d}\right\}} d \mu_{\gamma}^{k}(u)$. 
Hence, $\int f\left(\tilde{U}^{n}(t, u)\right) d \mu_{\gamma}(u)=\int f d \mu_{\gamma}$; on the other hand, denoting by $v^{n}$ the law of $\tilde{U}^{n}(\cdot, u)$, we also have:

$$
\begin{aligned}
\int f\left(\tilde{U}^{n}(t, u)\right) d \mu_{\gamma}(u) & =\int f\left((y(t)) d v^{n}(y)\right. \\
& =\int f\left(\tilde{U}^{\prime n}(t, \omega)\right) d P_{\gamma}(\omega) \\
& \stackrel{n \rightarrow \infty}{\longrightarrow} \int f(U(t, \omega)) d P_{\gamma}(\omega),
\end{aligned}
$$

which proves (ii). Let us now prove that $U(t, w)$ has values in the space $H^{1-\alpha}$. From (ii), we have:

$$
\begin{aligned}
\int \sum_{k} k^{2-2 \alpha}\left|U_{k}(t, \omega)\right|^{2} d P_{\gamma}(\omega) & =\int \sum_{k} k^{2-2 \alpha}\left|u_{k}\right|^{2} d \mu_{\gamma}(u) \\
& =\int\|u\|_{1-\alpha}^{2} d \mu_{\gamma}(u)<+\infty,
\end{aligned}
$$

therefore, $P_{\gamma}$ - a.e. $\omega, U(t, \omega) \in H^{1-\alpha}$.

It only remains to prove (i), i.e., that $U(t, \omega)$ is a flow associated with $B$. We have:

$$
\begin{aligned}
\int \mid \int_{0}^{t} & {\left[B_{k}^{n}\left(\tilde{U}^{\prime n}(s, \omega)\right)-B_{k}(U(s, \omega))\right] d s \mid d P_{\gamma}(\omega) } \\
\leqq & \int_{0}^{t}\left|B_{k}^{n}\left(\tilde{U}^{\prime n}(s, \omega)\right)-B_{k}\left(\tilde{U}^{\prime n}(s, \omega)\right)\right| d s d P_{\gamma}(\omega) \\
& \quad+\iint_{0}^{t}\left|B_{k}\left(\tilde{U}^{\prime n}(s, \omega)\right)-B_{k}(U(s, \omega))\right| d s d P_{\gamma}(\omega) .
\end{aligned}
$$

The second term converges towards zero by the equi-integrability of the functions $B_{k}^{n}\left(\tilde{U}^{n}(t, u)\right)$ and the fact that $\tilde{U}^{\prime n}(\cdot, \omega) \rightarrow U(\cdot, \omega)$ a.e. in the space $C\left(\mathbb{R}^{+} ; H^{1-\alpha}\right)$. The first term is equal to:

$$
\iint_{0}^{t}\left|B_{k}^{n}\left(\tilde{U}^{n}(s, u)\right)-B_{k}\left(\tilde{U}^{n}(s, u)\right)\right| d s d \mu_{\gamma}(u)
$$

and therefore, by the invariance of $\mu_{\gamma}$ under $\tilde{U}^{n}(0) \mapsto \tilde{U}^{n}(t)$ and the fact that $B_{k}^{n} \rightarrow B_{k}$ in $L^{2}\left(\mu_{\gamma}\right)$, this term converges to zero as well; therefore, and as we can identify in law the processes $\tilde{U}^{\prime n}(t, \omega)$ and the flows $\tilde{U}^{n}(t, u)$, we obtain (i) by passing to the limit.

Remark. By using a precompactness criterium due to Dubinski [Du], one can show that the laws $v^{n}$ of $U^{n}(\cdot, u)$ are precompact on the space

$$
Z=L_{\text {loc }}^{2}\left(\mathbb{R}^{+} ; H^{1-\alpha}\right) \cap C\left(\mathbb{R}^{+} ; H^{1-\beta}\right), \text { for } \frac{3}{2}<\alpha<\beta .
$$

In this way, and avoiding the use of the marginals $v_{k}$, we obtain a probability measure and realize a flow associated to $B$ on a single space, namely $Z$.

\section{The Perturbed Navier-Stokes Equation}

3.1. Invariant Measures. The classical Navier-Stokes equation describes the motion of an incompressible viscous fluid and is given by:

$$
\frac{\partial u}{\partial t}=-(u \cdot \nabla) u+\gamma \Delta u-\nabla p, \quad \operatorname{div} u=0
$$


where $\gamma>0$ is the viscosity coefficient. We will consider this equation, more precisely a stochastic perturbation of it, in the same context as for the Euler case (Sect. 1.2). This means we will consider (3.1) on the two-dimensional torus $\mathbb{T}^{2}$ and we will use the abstract Wiener space formulation of (1.3). We can then write the Navier-Stokes equation in the following form:

$$
\frac{d}{d t} u_{k}=B_{k}(u)-\gamma k^{2} u_{k} .
$$

As it is well known, this is no longer a conservative system (i.e. the energy is not conserved in time). One cannot therefore expect to have "good" invariant measures as in the Euler case. On the other hand, let us consider the following differential operator $Q$ :

$$
Q f(u)=\sum_{k} \frac{1}{k^{2}} D_{e_{k}}^{2} f(u)-\gamma k^{2} u_{k} D_{e_{k}} f(u), \quad f \in \mathscr{D} .
$$

This is an infinite dimensional Ornstein-Uhlenbeck operator of a type considered, e.g. in [Ga]. The measure $\mu_{\gamma}$ defined in (1.3) is an invariant measure for the Ornstein-Uhlenbeck flow generated by $Q$; in particular we have $\int Q f d \mu_{\nu}=0 \forall f \in \mathscr{D}$.

Let us consider the following operator:

$$
L f(u)=Q f(u)+\sum_{k} B_{k}(u) D_{e_{k}} f(u), \quad f \in \mathscr{D} .
$$

This can be regarded as the infinitesimal generator for a perturbed Navier-Stokes flow, as we show in the following paragraph. By the invariance of $\mu_{\gamma}$ under the Ornstein-Uhlenbeck flow and the invariance proved in Sect. 2 we have then in particular the infinitesimal invariance of the measure $\mu_{\gamma}$ under the Navier-Stokes flow in the sense that: $\int L f d \mu_{\gamma}=0 \forall f \in \mathscr{D}$.

3.2. The Perturbed Navier-Stokes Flow. Let $\mathbb{T} b_{t}=\sum_{k}(1 /|k|) b_{t}^{k} e_{k}$ be a normalized cylindric brownian motion on $H^{1}$, where the $b^{k}$ are independent copies of complex brownian motions. We recall that the Ornstein-Uhlenbeck process associated with $Q$ can be explicitly given by:

$$
\xi_{t}^{k}=x^{k} e^{-\gamma k^{2} t}+\int_{0}^{t} e^{-\gamma k^{2}(t-s)} \frac{\sqrt{2}}{|k|} d b^{k}(s)
$$

and that this process, starting from $H^{1-\alpha}$, actually remains in $H^{1-\alpha}$ for all times.

With respect to the existence of a perturbed Navier-Stokes flow, we have the following theorem (cf. [C1] for related results):

3.2.1. Theorem. There exists a stochastic process $x_{t} \in C\left(\mathbb{R}^{+} ; H^{1-\alpha}\right)$, such that, for $x \in H^{1-\alpha}$, and writing $x_{t}=\sum x_{t}^{k} e_{k}$, we have:

$$
x_{t}^{k}=x^{k}+\tilde{\pi} \pi b_{t}^{k}-\int_{0}^{t}\left[\gamma k^{2} x_{s}^{k}-B_{k}\left(x_{s}\right)\right] d s \quad \text { a.e., } \quad \forall t \in \mathbb{R}^{+},
$$

where $\tilde{\pi} b_{t}$ is a Brownian motion on $H^{1}$.

Moreover, $\mu_{\gamma}$ is invariant for $x_{t}$ in the sense that

$$
\int E_{x} f\left(x_{t}\right) d \mu_{\gamma}(x)=\int f d \mu_{\gamma} \quad \forall t \in \mathbb{R}^{+}, \quad \forall f \in \mathscr{D} .
$$


Proof. Let us consider the finite dimensional approximations of $B_{k}$ defined in (1.5), (2.1), and, for $x \in H^{1-\alpha}$, put $x^{n}=\left(x^{n, \alpha_{1}}, \ldots, x^{n, \alpha_{d}}\right)$, where clearly $d$ depends on $n$. Since the coefficients are regular, we have, by classical results, the existence of a finite dimensional stochastic process $x_{t}^{n}=\left(x_{t}^{n, \alpha_{1}}, \ldots, x_{t}^{n, \alpha_{d}}\right) \in \mathbb{C}^{d}$ defined for small times and satisfying:

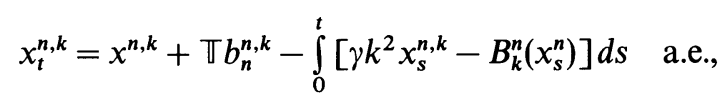

where $b_{t}^{n}=\sum_{k \in\left\{\alpha_{1}, \ldots, \alpha_{d}\right\}} b_{t}^{k}(1 /|k|) e_{k}$. By Itô’s formula, we have

$$
\begin{aligned}
d\left|x_{t}^{n, k}\right|^{2}= & \frac{2 \sqrt{2}}{|k|}\left(\operatorname{Re}\left(x_{t}^{n, k}\right) d \operatorname{Re}\left(b_{t}^{n, k}\right)+\operatorname{Im}\left(x_{t}^{n, k}\right) d \operatorname{Im}\left(b_{t}^{n, k}\right)\right) \\
& -\left[2 \gamma k^{2}\left|x_{t}^{n, k}\right|^{2}-\frac{2}{k^{2}}-2 \operatorname{Re}\left(B_{k}^{n}\left(x_{t}^{n}\right) \cdot \bar{x}_{t}^{n, k}\right)\right] d t .
\end{aligned}
$$

But, because of the invariance of the energy (cf. 1.2), we have $\sum k^{2} B_{k}^{n}(x) \cdot \bar{x}_{k}=0$ and therefore,

$$
E\left(\left\|x_{t}^{n}\right\|_{1}^{2}\right)=E\left(\sum_{k} k^{2}\left|x_{t}^{n}\right|^{2}\right)=\left\|x^{n}\right\|_{1}^{2}-2 \gamma \int_{0}^{t}\left\|x_{s}^{n}\right\|_{2}^{2} d s+c(n) t .
$$

Moreover

$$
\begin{aligned}
& E\left(\sup _{t \in[0, T]}\left\|x_{t}^{n}\right\|_{1}^{2}\right) \leqq\left\|x^{n}\right\|_{1}^{2}+c(n) T \\
& +8 E \sup \sum_{k} \int_{0}^{t}\left|\left(\operatorname{Re}\left(x_{s}^{n, k}\right) d \operatorname{Re}\left(b_{s}^{n, k}\right)+\operatorname{Im}\left(x_{s}^{n, k}\right) d \operatorname{Im}\left(b_{s}^{n, k}\right)\right)\right|^{2} \\
& \leqq\left\|x^{n}\right\|_{1}^{2}+c(n) T+c E \int_{0}^{T} \sum_{k}\left|x_{s}^{n, k}\right|^{2} d s \\
& \leqq\left\|x^{n}\right\|_{1}^{2}+T\left(\left\|x^{n}\right\|_{0}^{2}+c^{\prime}(n)\right) \text {, }
\end{aligned}
$$

where we used (3.5) in the last inequality. This estimation implies the non-explosion in finite time of the processes $x_{t}^{n}$, for fixed $n$.

We have $\int E_{x} f\left(x_{t}^{n}\right) d \mu_{y}(x)=\int f d \mu_{y} \forall t>0, f \in \mathscr{D}$, by the invariance of the measure under the finite dimensional flow $x_{t}^{n}$.

Let $\rho^{n, k}$ denote the law of $x_{t}^{n, k}$ on the space $C\left(\mathbb{R}^{+} ; \mathbb{C}\right)$. We will apply the precompactness criterium (2.4) in order to get a weak limit of these measures. We have:

$$
\begin{gathered}
\rho^{n, k}(|y(0)|>R) \leqq \frac{1}{R}\left|x^{k}\right| \leqq \frac{c}{R} \quad \forall n, \\
\rho^{n, k}\left(\sup _{\substack{0 \leq t \leq t^{\prime} \leqq T \\
t^{\prime}-t \leqq \delta}}\left|y(t)-y\left(t^{\prime}\right)\right| \geqq \rho\right) \leqq \frac{1}{\rho} E \sup \left|x_{t}^{n, k}-x_{t^{\prime}}^{n, k}\right| \\
\leqq \frac{c}{\rho}\left(\delta^{\alpha}+\gamma k^{2} E \sup \int_{t}^{t^{\prime}}\left|x_{s}^{n, k}\right| d s+E \sup \int_{t}^{t^{\prime}}\left|B_{k}^{n}\left(x_{s}^{n}\right)\right| d s\right),
\end{gathered}
$$


with $0<\alpha<\frac{1}{2}$, by the properties of brownian motion and Eq. (3.4). Using Hölder inequality and the initial condition, we have:

$$
\begin{aligned}
E\left(\sup \int_{t}^{t^{\prime}}\left|x_{s}^{n, k}\right| d s\right) & \leqq \delta^{1 / 2}\left(E \int_{0}^{T}\left|x_{s}^{n, k}\right|^{2} d s\right)^{1 / 2} \\
& \leqq \delta^{1 / 2}\left(\int_{0}^{T}\left(\left|x^{k}\right|^{2}+\frac{2 s}{k^{2}}\right) d s\right)^{1 / 2} \\
& \leqq \delta^{1 / 2}\left(\left|x^{k}\right|^{2} T+\frac{T^{2}}{k^{2}}\right)^{1 / 2} \quad \forall n ;
\end{aligned}
$$

moreover, using again the invariance of $\mu_{\gamma}$ under $x_{t}^{n}$,

$$
\begin{aligned}
E\left(\sup \int_{t}^{t^{\prime}}\left|B_{k}^{n}\left(x_{s}^{n}\right)\right| d s\right) & \leqq \delta^{1 / 2}\left(E\left(\int_{0}^{T}\left|B_{k}^{n}\left(x_{s}^{n}\right)\right|^{2} d s\right)\right)^{1 / 2} \\
& \leqq(\delta T)^{1 / 2}\left(\int\left|B_{k}^{n}(x)\right|^{2} d \mu_{\gamma}(x)\right)^{1 / 2}
\end{aligned}
$$

this last integral being uniformly bounded in $n$ by the estimations of Lemma 1.3.2.

Hence we will have a subsequence $\rho^{n_{j}, k}$ converging towards $\rho^{k}$ and $\rho^{k}$ will be the law of a process $x_{t}^{k} \in C\left(\mathbb{R}^{+} ; \mathbb{C}\right)$, by Skorohod's theorem. Let $x_{t}=\sum x_{t}^{k} e_{k}$. We have

$$
\begin{aligned}
E\left(\left\|x_{t}\right\|_{1-\alpha}^{2}\right) & =E\left(\sum_{k} k^{2-2 \alpha}\left|x_{t}^{k}\right|^{2}\right)=\lim _{n_{j}} \sum_{k^{2} \leqq n_{j}} k^{2-2 \alpha} E\left(\left|x_{t}^{n_{j}, k}\right|^{2}\right) \\
& \leqq \sum_{k} k^{2-2 \alpha} \int\left|x_{k}\right|^{2} d \mu_{\gamma}(x)=\int\|x\|_{1-\alpha}^{2} d \mu_{\gamma}(x)<+\infty,
\end{aligned}
$$

and therefore the process lives a.s. in $H^{1-\alpha}$.

The relation (ii) follows from the analogous relation for the finite dimensional approximations. It only remains to check that $x_{t}^{k}$ satisfies (i), but this can be done by taking limits in (3.4), remembering that $x_{t}^{n_{j}, k} \rightarrow x_{t}^{k}$ a.e., uniformly with respect to $t$, and also that the $B_{k}$ are equi-integrable in $L^{2}\left(\mu_{\gamma}\right)$.

Acknowledgements. The second author wishes to express her gratitude to Professor Paul Malliavin for very helpful discussions. She also would like to thank the Université de Paris VI and the Ruhr University of Bochum for their warm hospitality. Both authors are also grateful to Prof. Raphael H申egh-Krohn for very stimulating discussions and to Prof. David Elworthy and the Institute of Mathematics, University of Warwick for kind invitations. They are also indebted to Prof. Shigeo Kusuoka and Dr. Walter Schneider for a critical reading of the manuscript and stimulating discussions. Partial financial support of the Volkswagenstiftung and the SFB 237 Bochum Essen Düsseldorf is gratefully acknowledged.

Dedication. After completion of this work the terrible news of the sudden death of Raphael $\mathrm{H} \phi \mathrm{egh}-\mathrm{Krohn}$ reached us. In deep sorrow we mourn his departure. The present work has its roots in previous inspiring work by him and we dedicate it to him as a small sign of our gratitude.

\section{References}

[AHK1] Albeverio, S., H $\phi$ egh Krohn, R.: Stochastic flows with stationary distribution for two-dimensional inviscid fluids. Stoch. Proc. Appl. 31, 1-31 (1989)

[AHK2] Albeverio, S., H $\phi$ egh-Krohn, R.: Stochastic methods in quantum field theory and hydrodynamics. Phys. Repts. 77, 193-214 (1981) 
[AHKDeF] Albeverio, S., Ribeiro de Faria, M., Hфegh Krohn, R.: Stationary measures for the periodic Euler flow in two dimensions. J. Stat. Phys. 20, 585-595 (1979)

[AHKM] Albeverio, S., H $\phi$ egh-Krohn, R., Merlini, D.: Some remarks on Euler flows, associated generalized random fields and Coulomb systems, pp. 216-244. In: Infinite dimensional analysis and stochastic processes. Albeverio, S. (ed.). London: Pitman 1985

[BF1] Boldrighini, C., Frigio, S.: Equilibrium states for the two-dimensional incompressible Euler fluid. Atti Sem. Mat. Fis. Univ. Modena XXVII, 106-125 (1978)

[BF2] Boldrighini, C., Frigio, S.: Equilibrium states for a plane incompressible perfect fluid. Commun. Math. Phys. 72, 55-76 (1980); Errata: ibid. 78, 303 (1980)

[BPP] Benfatto, G., Picco, P., Pulvirenti, M.: On the invariant measures for the two-dimensional Euler flow, CPT-CNRS Preprint, Marseille-Luminy (1986)

[C1] Cruzeiro, A. B.: Solutions et measures invariants pour des équations d'évolution stochastiques du type Navier-Stokes. Expositiones Mathematicae 7, 73-82 (1989)

[C2] Cruzeiro, A. B.: to appear in Proc. 1987 Delphis-Conf. (1988)

[C3] Cruzeiro, A. B.: Equations Différentielles sur l'espace de Wiener et formules de CameronMartin non-linéaires. J. Funct. Anal. 54, 206-227 (1983)

[CDG] Caprino, S., De Gregorio, S.: On the statistical solutions of the two-dimensional periodic Euler equations. Math. Methods Appl. Sci. 7, 55-73 (1985)

[DeF] Ribeiro de Faria, M.: Fluido de Euler bidimensional: construção de medidas estacionárias e fluxo estocástico. Diss., Universidade do Minho, Braga (Portugal), 1986

[DP] Dürr, D., Pulvirenti, M.: On the vortex flow in bounded domain. Commun. Math. Phys. 83, 265-273 (1983)

[Du] Dubinskii, Y.: Weak convergence for nonlinear elliptic and parabolic equations. Mat. Sb. 67, 609-642 (1965) (Russ.)

[E] Ebin, D. G.: A concise presentation of the Euler equations of hydrodynamics. Commun. Partial Diff. Equations 9, 539-559 (1984)

[FT] Foias, C., Temam, R.: Self-similar universal homogeneous statistical solutions of the Navier-Stokes equations. Commun. Math. Phys. 90, 187-206 (1983)

[G] Glaz, H.: Statistical behavior and coherent structures in two-dimensional inviscid turbulence. Siam J. Appl. Math. 41, 459-479 (1981)

[Ga] Gaveau, B.: Noyau de probabilités de transition de certains opérateurs d'OrnsteinUhlenbeck dans l'espace de Hilbert. C.R. Acad. Sci. Paris, Ser. I, 293, 469-472 (1981)

[IW] Ikeda, N., Watanabe, S.: Stochastic differential equations and diffusion processes. Amsterdam: North-Holland 1981

[K] Kuo, H. H.: Gaussian Measures in Banach Spaces. Lecture Notes in Mathematics, vol. 463. Berlin, Heidelberg, New York: Springer 1979

[KK] Krée, M., Krée, P.: Continuité de la divergence dans les espaces de Sobolev relatifs à l'espace de Wiener. C.R. Acad. Sci. Paris, Ser. I, (1983)

[KrMo] Kraichnan, R. H., Montgomery, D.: Two-dimensional turbulence. Rep. Progr. Phys. 43, 547-619 (1980)

[Ma] Malliavin, P.: Implicit functions in finite corank on the Wiener space. In: Taniguchi Symposium 1982. Tokyo: Kinokuniya 1984

[MP] Marchioro, C., Pulvirenti, M.: Vortex methods in two-dimensional fluid mechanics. Lecture Notes in Physics, vol. 203. Berlin, Heidelberg, New York: Springer 1984

[SV] Stroock, D. W., Varadhan, S. R. S.: Multidimensional diffusion processes. Grundlehren Mathem. vol. 233. Berlin, Heidelberg, New York: Springer 1979

[Te] Temam, R.: Navier-Stockes equations; theory and numerical analysis. Amsterdam: North-Holland 1977

[VKF] Vishik, M. I., Komechi, A. I., Fursikov, A. V.: Some mathematical problems of statistica! hydrodynamics. Russ. Math. Surv. 34, 149-234 (1979)

[W] Welz, B.: Stochastische Gleichgewichtsverteilung eines 2-dimensionalen Superfluids. Diplomarbeit, Bochum (1986)

Communicated by H. Araki

Received May, 20, 1988; in revised form June 2, 1989 